Exp. Anim. 52(1), 81-84, 2003

\title{
Differences between BALB/c and C57BL/6 Mice in Mouse Hepatitis Virus Replication in Primary Hepatocyte Culture
}

\author{
Shigeru KYUWA1), Seiji KAWAMURA2), Yoh-ichi TAGAWA ${ }^{1)}$, \\ Yoichiroh IWAKURA ${ }^{1)}$, Toru URANO3), and Yasuhiro YOSHIKAWA ${ }^{2)}$
}

\begin{abstract}
1) Center for Experimental Medicine, Institute of Medical Science, The University of Tokyo, 4-6-1 Shirokanedai Tokyo 108-8639, 2)Department of Biomedical Science, Graduate School of Agricultural and Life Sciences, The University of Tokyo, 1-1-1 Yayoi, Bunkyo-ku, Tokyo 113-8657, ${ }^{3)}$ Division of Microbiology and Genetics, Center for Animal Resources and Development, Kumamoto University, 2-2-1 Honjo Kumamoto 860-0811, Japan
\end{abstract}

\begin{abstract}
We previously showed that an intraperitoneal infection with mouse hepatitis virus $(M H V)$ resulted in acute hepatic failure accompanying extremely elevated viral growth in the liver in interferon- $\gamma$-deficient BALB/C (BALB-GKO), but not C57BL/6 (B6-GKO) mice. To examine the basis of the strain difference against $M H V$ infection in interferon- $\gamma$-deficient mice, viral replication in primary hepatocyte cultures from $B A L B / C$ and $B 6$ mice with or without the IFN- $\gamma$ gene was compared in vitro. The MHV replication in BALB/C hepatocytes with or without the IFN- $\gamma$ gene was significantly higher than that in B6 hepatocytes with or without the IFN- $\gamma$ gene, suggesting that there is a strain difference in MHV replication in hepatocytes. Since a significant difference in MHV replication in hepatocytes was not observed between wild type and IFN- $\gamma$-deficient mice of the same genetic background, the phenomenon is thought to be independent of IFN- $\gamma$. However, pretreatment of hepatocytes with recombinant mouse interferon- $\gamma$ inhibited MHV replication in a dose-dependent fashion. The results are discussed with respect to the pathology of MHV infection in mice with or without the IFN- $\gamma$ gene.
\end{abstract}

Key words: mouse hepatitis virus, hepatocyte, viral replication

Viral replication in vivo is generally regulated by intrinsic as well as extrinsic factors. The intrinsic factors reside in virus-infected cells and some of them are directly involved in viral replication. On the other hand, the extrinsic factors exist outside of virus-infected cells and regulate viral replication indirectly. They involve virus-specific antibodies, cytotoxic $\mathrm{T}$ lymphocytes and other elements in the immune system and extend throughout most of the body. It is generally difficult to evaluate how the intrinsic factors play a role in viral replication in vivo. Reciprocally, we can evaluate them, if host cells are infected with virus in the absence of the immune system.

Mouse hepatitis virus (MHV) infection is an impor-

(Received 13 June 2002 / Accepted 18 September 2002)

Address corresponding: S. Kyuwa, Department of Biomedical Science, Graduate School of Agricultural and Life Sciences, The University of Tokyo, 1-1-1 Yayoi, Bunkyo-ku, Tokyo 113-8657, Japan 
tant viral infection in contemporary laboratory mouse colonies $[4,6,15]$. Recently, we have described that interferon- $\gamma($ IFN- $\gamma$ ) modifies MHV infection in mice $[7,8]$. MHV replicates well in the liver and induces acute hepatic failure in IFN- $\gamma$-deficient (GKO) BALB/ c (BALB-GKO) mice, whereas IFN- $\gamma$-deficient C57BL/ 6 (B6-GKO) mice show milder pathological changes with lower viral growth in the liver after intraperitoneal MHV infection. To understand the mouse strain difference against MHV infection in GKO mice, we set up an in vitro system to quantify the viral replication in hepatocytes in a condition possibly not restricted by the immune system, and compared viral replication in hepatocytes from BALB/c and B6 mice with or without the IFN- $\gamma$ gene.

The GKO and wild type (WT) mice of BALB/c and B6 backgrounds, and JHMV used in this study were described previously [7, 8, 12]. Mice free of MHV were used throughout the experiments. The viral titer was determined by plaque assayed on DBT cells [8]. The experiments were conducted according to institutional ethical guidelines for animal experiments and safety guidelines for gene manipulation experiments.

To establish a primary culture of hepatocytes in vitro, hepatocytes were isolated from mice after an in situ perfusion with collagenase solution as described elsewhere $[1,11,13]$. The hepatocyte preparation was washed by centrifugation at $30 \times \mathrm{g}$ for $90 \mathrm{~s}$ four or five times with William's medium E supplemented with $10^{-7}$ $\mathrm{M}$ insulin, $10^{-7} \mathrm{M}$ dexamethasone and $50 \mu \mathrm{g} / \mathrm{ml}$ kanamycin. The hepatocytes were seeded in 24-well plates at a concentration of $4 \times 10^{5}$ cells $/ \mathrm{ml}$ in $0.3 \mathrm{ml}$ of William's medium E supplemented with 5\% fetal calf serum. After incubation at $37^{\circ} \mathrm{C}$ for $2 \mathrm{~h}$, the culture medium was changed to fresh William's medium $\mathrm{E}$ and then cultured overnight. The culture was infected with JHMV at a multiplicity of infection (m.o.i.) of 0.1, washed twice and then cultured. The residual virus after washing was less than 25 plaque-forming units $(\mathrm{PFU}) / \mathrm{ml}$ (data not shown). After incubation at $37^{\circ} \mathrm{C}$ for 12 and $24 \mathrm{~h}$, the culture supernatants were harvested, and assayed for their viral titer as described above.

Evaluation of statistical differences between data was assessed for statistical significance by Student's $t$-test or the log-rank test. A difference was considered statistically significant at $P<0.05$.

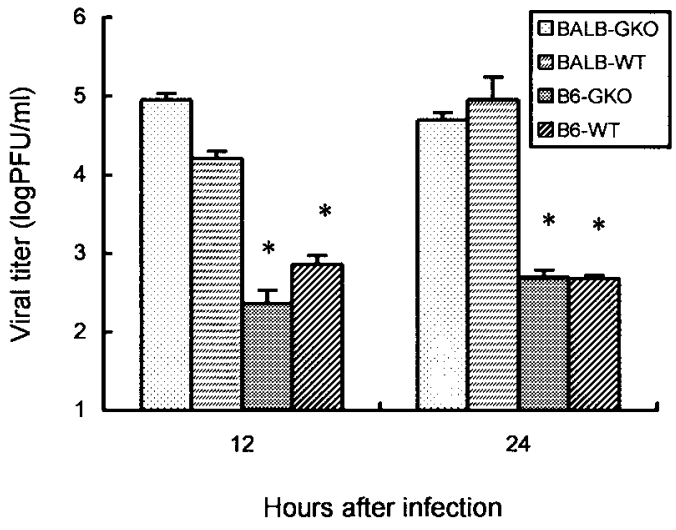

Fig. 1. JHMV replication in primary hepatocyte cultures from BALB-GKO, BALB-WT, B6-GKO and B6-WT mice in vitro. The cells were infected with JHMV at an m.o.i of 0.1 and the viral titers in the supernatant at 12 and $24 \mathrm{~h}$ post-infection were determined by plaque assay. Data are expressed as mean + standard deviation. Substantially same results were obtained in another experiment. *: $P<0.05 \mathrm{~B} 6-\mathrm{GKO}$ versus BALB-GKO, and B6-WT versus BALB-WT.

The primary culture of hepatocytes was prepared from WT and GKO mice of BALB/c as well as those of a B6 background. They were infected with JHMV, and the viral titer of the culture supernatant was determined at 12 and $24 \mathrm{~h}$ post-infection (Fig. 1). The viral titer of BALB-GKO mice was 390 and 100 folds higher than that of B6-GKO mice at 12 and $24 \mathrm{~h}$ post-infection, respectively, indicating that JHMV replication in BALB-GKO was higher than that in B6-GKO mice even in the absence of the immune system. Surprisingly, the viral titer of BALB-WT mice was 20 and 190 folds higher than that of B6-WT mice at 12 and 24 $\mathrm{h}$ post-infection, respectively. There was no significant difference in MHV replication between WT and GKO mice of the same genetic background. These results suggest that the phenomenon is not dependent on IFN$\gamma$ and that the viral replication in the hepatocytes with a BALB/c background is significantly higher than in those with a B6 background. Microscopic examination of infected primary hepatocytes revealed that JHMV induced a multinucleated giant cell formation in all the samples (data not shown), as described previously [13].

To examine the role of IFN- $\gamma$ in JHMV replication in primary hepatocyte culture, the cells were incubated with $100,10,1 \mathrm{U} / \mathrm{ml}$ of recombinant mouse IFN- $\gamma$ (a 
(A)

BALB-GKO

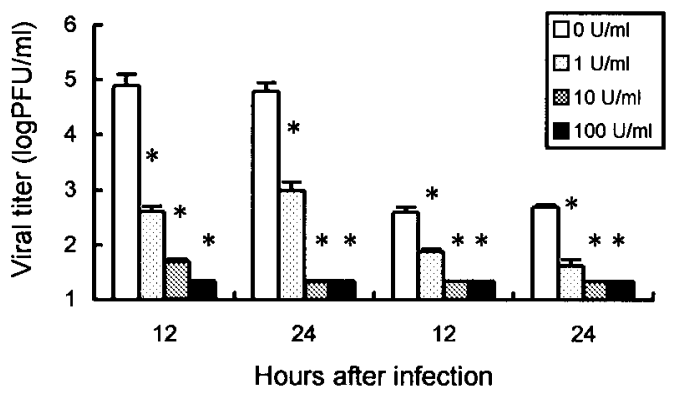

(B)

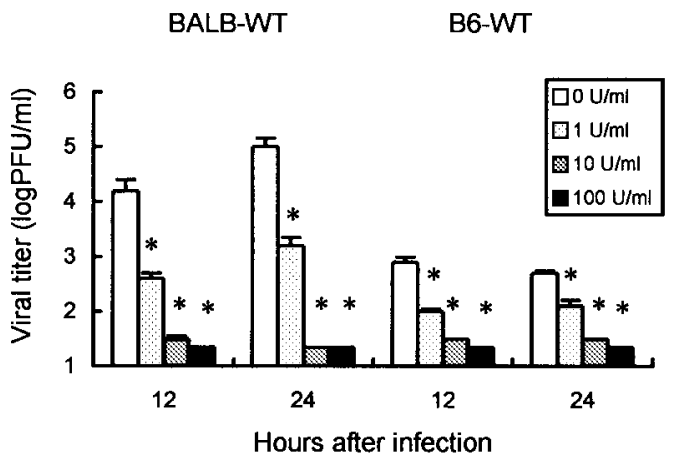

Fig. 2. Effect of recombinant IFN- $\gamma$ pretreatment on JHMV replication in primary hepatocytes from BALB-GKO and B6-GKO (A), and BALB$\mathrm{WT}$ and $\mathrm{B} 6-\mathrm{WT}$ (B) mice in vitro. The cells with or without IFN- $\gamma$ pretreatment were infected with JHMV at an m.o.i. of 0.1 and the viral titers in the supernatant at 12 and $24 \mathrm{~h}$ post-infection were determined by plaque assay. Data are expressed as mean + standard deviation. *: $P<0.05$ versus non-treated group.

kind gift from Shionogi, Osaka, Japan) and medium alone overnight, and then infected with JHMV as described above. Pretreatment with mouse IFN- $\gamma$ clearly inhibited viral replication in primary hepatocyte cultures in a dose dependent fashion (Fig. 2A), and $100 \mathrm{U} /$ $\mathrm{ml}$ of IFN- $\gamma$ completely inhibited the viral replication in primary hepatocyte cultures from both BALB-GKO and B6-GKO mice. Similar inhibition of viral replication was also observed in primary hepatocytes from BALB-WT and B6-WT mice (Fig. 2B), demonstrating that IFN- $\gamma$ can inhibit JHMV replication in hepatocytes from BALB-GKO, B6-GKO, BALB-WT and B6-WT mice.
In the present study, we found that JHMV replication in hepatocytes from BALB/c background mice was significantly higher than in those from B6 background mice. The result implies that some intrinsic factor(s) in $\mathrm{BALB} / \mathrm{c}$ hepatocytes, which remain to be elucidated, facilitate MHV replication more effectively than in B6 hepatocytes. Cellular receptor for MHV (Ceacam 1) is one of the known intrinsic factors that regulate MHV replication [14]. Recent studies reported that the genotype of Ceacam 1 gene is correlated with the susceptibility to JHMV [9], and that targeted disruption of the Ceacam 1 gene leads to reduced susceptibility of mice to MHV infection [2]. Although BALB/c and B6 mice are reported to have the same genotype [9], we do not know whether the density of this protein on BALB/ $\mathrm{c}$ hepatocytes is higher than that on B6 hepatocytes. It therefore remains to be tested whether this molecule explains the strain difference in JHMV replication in hepatocytes. Of course, we cannot exclude the possibility that other unknown factor(s) play an essential role in the strain difference in MHV replication in hepatocytes.

There was no significant difference in MHV replication in hepatocytes between WT and GKO mice. However, pretreatment of hepatocytes with recombinant mouse IFN- $\gamma$ inhibited MHV replication in a dose-dependent fashion. A simplistic view is that these results are inconsistent. There is no doubt that endogenous as well as exogenous mouse IFN- $\gamma$ can work in hepatocytes [8]. The most likely explanation is that the primary hepatocyte cultures we used in the present study contained few IFN- $\gamma$ producing cells such as T, NK and NKT cells [3], or produced little IFN- $\gamma$ during the experiment even if a small number of IFN- $\gamma$ producing cells are present in the cultures.

We previously reported viral growth in the liver in IFN- $\gamma^{-/}$and IFN- $\gamma^{+/-}$mice of either BALB/c or B6 background after intraperitoneal JHMV infection [8]. In this study, we report JHMV replication in primary hepatocyte culture from the same strain of mice in vitro. From these data, the effect of mouse genetic background and IFN- $\gamma$ on MHV replication in hepatocytes/liver in in vitro and in vivo systems is summarized in Table 1. First, we are aware that the profile of viral replication in the in vitro system bears a resemblance to that in the in vivo system. It may indicate that there is a correlation between the in vitro and in vivo systems. In this 
Table 1. Effect of mouse genetic background and IFN- $\gamma$ on MHV replication in hepatocytes in vitro and viral titer in the liver in MHV-infected mice

\begin{tabular}{lcclcc}
\hline \multirow{2}{*}{$\begin{array}{l}\text { Background } \\
\text { of mice }\end{array}$} & \multicolumn{3}{c}{ Viral replication } & in hepatocytes/liver \\
\cline { 2 - 3 } \cline { 5 - 6 } \cline { 5 - 6 } & without IFN- $\gamma$ & with IFN- & & without IFN- $\gamma$ & with IFN- $\gamma$ \\
\cline { 2 - 3 } BALB/c & +++++ & +++ & & +++++ & ++++ \\
B6 & +++ & ++ & & ++++ & +++ \\
\hline
\end{tabular}

a) The number of symbol + corresponds viral titer $(\log \mathrm{PFU} / \mathrm{ml})$ at $24 \mathrm{~h}$ post-infection in hepatocytes from BALB-GKO and B6-GKO with or without pretreatment of recombinant IFN- $\gamma(1 \mathrm{U} / \mathrm{ml})$ in Fig. 2 . b) The number of symbol + corresponds viral titer $(\log \mathrm{PFU} / \mathrm{g})$ in the liver in IFN- $\gamma^{-/-}$and IFN- $\gamma^{+/-}$mice at 3 days post-infection [8].

study, we found that JHMV replication in hepatocytes from $\mathrm{BALB} / \mathrm{c}$ background mice was significantly higher than in those from B6 background mice using an in vitro system. It is likely that the same event occurs in vivo. Namely, JHMV replication in BALB/c hepatocytes in vivo may be higher than that in B6 hepatocytes in vivo. This may be one reason why BALB-GKO mice die after intraperitoneal JHMV infection whereas the disease in B6-GKO mice is mild. On the other hand, IFN- $\gamma$ suppresses JHMV replication in hepatocytes. A large difference between BALB/c and B6 mice in JHMV replication in hepatocytes in vitro was obscured by the pretreatment of IFN- $\gamma$, which inhibited viral replication in hepatocytes from both strains. This might also occur in the in vivo system. Although JHMV replication in hepatocytes in BALB-WT in vivo may be naturally higher than in those in B6-WT mice in vivo, the difference in JHMV replication might be lessened by IFN- $\gamma$ produced by T, NK and NKT cells in WT mice.

Of course, we cannot exclude the possibility that other factors are also involved in the distinct profiles of JHMV replication in vivo. It is well known that BALB/ $\mathrm{c}$ and B6 mice have the distinct allele of the major histcompatibility complex genes, which plays a critical role in $\mathrm{T}$ cell-mediated immune responses $[5,10]$. To elucidate the role, comparative studies with BALB-GKO mice and BALB-GKO with the $\mathrm{H}-2^{\mathrm{b}}$ haplotype, the same $\mathrm{H}-2$ haplotype as in B6-GKO mice, are in progress.

\section{References}

1. Arnheiter, H., Baechi, T., and Haller, O. 1982. J. Immunol. 29: 1275-1281.

2. Blau, D.M., Turbide, C., Tremblay, M., Olson, M., Letourneau, S., Michaliszyn, E., Jothy, S., Holmes, K.V., and Beauchemin, N. 2001. J. Virol. 75: 8173-8186.

3. Billiau, A., Heremans, H., Vermeire, K., and Matthys, P. 1998. Ann. N. Y. Acad. Sci. 856: 22-32.

4. Compton, S.R., Barthold, S.W., and Smith. A.L. 1993. Lab. Anim. Sci. 43: 15-28.

5. Gonzalez-Gay, M.A., Zanelli, E., Khare, S.D., Krco, C.J., Griffiths, M.M., Luthra, H.S., and David, C.S. 1996. Immunogenet. 44: 377-384.,

6. Homberger, F. R. 1997. Lab. Anim. 31: 97-115.

7. Kyuwa, S., Tagawa, Y., Shibata, S., Doi, K., Machii, K., and Iwakura, Y. 1998. J. Virol. 72: 9286-9290.

8. Kyuwa, S., Kawamura, S., Shibata, S., Machii, K., Tagawa, Y., Iwakura, Y., and Urano, T. 2002. Virus Res. 83: 169177.

9. Ohtsuka, N. and Taguchi, F. 1997. J. Virol. 71: 88608863.

10. Potter, M.R., Noben-Trauth, N., Weis, J. H., Teuscher, C., and Weis, J.J. 2000. Infect. Immun. 68: 5603-5609.

11. Seglen, P.O. 1976. Methods Cell. Biol. 13: 29-81.

12. Tagawa, Y., Sekikawa, K., and Iwakura, Y. 1997. J. Immunol. 159: 1418-1428.

13. Taguchi, F., Kawamura, S., and Fujiwara, K. 1983. Infect. Immun. 39: 955-959.

14. Williams, R.K., Jiang, G.S., and Holmes, K.V. 1991. Proc. Natl. Acad. Sci. U.S.A. 88: 5533-5536.

15. Yamada, Y.K. and Yabe, M. 2000. Exp. Anim. 49: 61-66. 